\title{
AnyPLACE - An Energy Management System to Enhance Demand Response Participation
}

\author{
C. Abreu, D. Rua, T. Costa, P. Machado \\ INESC TEC \\ Porto, Portugal \\ \{crabreu, drua, tacosta, paam\}@inesctec.pt
}

\author{
J. A. Peças Lopes \\ FEUP, INESC TEC \\ Porto, Portugal \\ jpl@fe.up.pt
}

\author{
M. Heleno \\ Lawrence Berkeley National Laboratory \\ Berkeley, United States \\ miguelheleno@1nl.gov
}

\begin{abstract}
This paper describes an energy management system that is being developed in the AnyPLACE project to support new energy services, like demand response, in residential buildings. In the project end-user interfaces are designed and implemented to allow the input of preferences regarding the flexible use of shiftable and thermal appliances. Monitoring and self-learning algorithm are used to allow additional information to be collected and an automation platform is available for the management and control of appliances. An energy management algorithm is presented that processes end-user preferences and devices characteristics to produce an optimal dispatch considering demand response incentives. Results show the successful implementation of an optimized energy scheduling.
\end{abstract}

Index Terms-optimization; energy management; user interfaces; home automation.

\section{INTRODUCTION}

The advent of the smart grid has been used to explore new energy services towards the inclusion of new stakeholders. Many studies have developed strategies showing that retailers and aggregators can benefit from a combined participation of different consumers in Demand Response (DR) programs by increasing their remuneration form the demand side bidding in energy and ancillary services markets, e.g. [1][2]. However, after decades of scientific contributions on load management and control, aggregation methods, bidding strategies, economic and studies and pilot projects on the field, the massive participation of DR programs by residential consumers is far from being a reality. Thus, the engagement of end-users is still a major challenge towards the deployment of domestic Energy Management Systems (EMS) and it can be divided in three main dimension: (1) the development of efficient load control algorithms that enable the optimal use of resources in scenarios of price differentiation (e.g., variable tariffs) and the integration of microgeneration at the building level; (2) the conception of innovative human-machine interaction approaches capable of influencing consumption habits through energy awareness and incentivizing end-users to abandon their passive role and become active participants in managing their energy uses; (3) the adoption of efficient automation and ICT infrastructures, interoperable with the different systems available in the domestic environment (Wi-Fi network, smart appliances, gadgets, etc.), creating the conditions for the provision of a variety of new energy services both to the end-users and the grid.

The fundamental contribution of the AnyPLACE project is to combine these three dimensions in a platform. Based on existing single board computing systems, like the Raspberry PI, to ensure a cost-effective approach an EMS is being created considering a different perspective on end-user engagement, where the energy management algorithms, the design of human-machine interactions and the ICT infrastructure are viewed in an integrated manner. This paper aims at discussing some aspects of this vision and presenting exploratory results of the project regarding the optimal use of appliances in residential buildings. The paper is divided as follows: section II presents the rationale for the user interface development used in the design of end-user interfaces; section III defines the formulation of the energy management algorithm being implemented in the AnyPLACE solution for optimal scheduling of devices and systems; section IV discusses the automation implementation that allow the implementation of the optimal schedule using a modular system to support the necessary data exchange; section $\mathrm{V}$ presents the main considerations about the work produced and described in this paper.

\section{USER INTERFACES DESIGN}

User interface design for home automation systems has come a long way from primitive designs presented on earlier iterations of such systems. Mobile computing, with devices such as smartphones or tablets, has given users access to rich user centric interfaces, capable of showing several information in real-time. However, simplicity must be the main guideline for any Graphical User Interface (GUI) design, so that users are not overloaded with unnecessary data. Also, the displaying of relevant data needs to make use of strategies that extract information in an automated and preferably non-intrusive manner (or minimally intrusive).

The user interface layer should be separated from underlying data and communication layers, used to interact 
with other integrated modules on the system. Previous works on this subject have shown concerns that should be present on GUI design, like in [3], where energy efficiency of GUI on devices is explored and several techniques used to improve both energy consumption and user performance. GUI design for demanding environments is the main topic in [4]. Specific constraints, such as limited screen sizes are considered as design guidelines for the user interface. Modularity is also addressed in this work, relying on a modified Model-viewcontroller (MVC) software pattern for layer separation. In [5] a context-sensitive user interface is presented, capable of showing personalized information, based on the user preferences.

As such, AnyPLACE GUI has the following requirements: usage on different screen sizes, ability to control all parameters of the solution and dynamic presentation of relevant data. The AnyPLACE solution relies on computational module with an optional local display and it is capable of interacting with mobile display systems (e.g. smartphones, tablets, etc,). Hence a unified GUI was created to be dynamically adaptable for both types of display. A "dashboard" approach was used for the AnyPLACE platform, where the total area of each screen is divided into various interactive blocks, grouped by its relevance or area. With this approach, no screen region is wasted and all information is presented to the users, using a modern and responsive user centric design. PhoneGap [6], was used as a web-based mobile development framework. Like the AnyPLACE local display GUI, the smartphone applications are based on HTML5, CSS and Javascript. This web-based content is embedded into native smartphone applications, without resorting to any platform-dependent coding.

Fig.1 shows two sample screens from the AnyPLACE smartphone application: the first screen is related to the user preferences and the second screen graphically represents metering data.

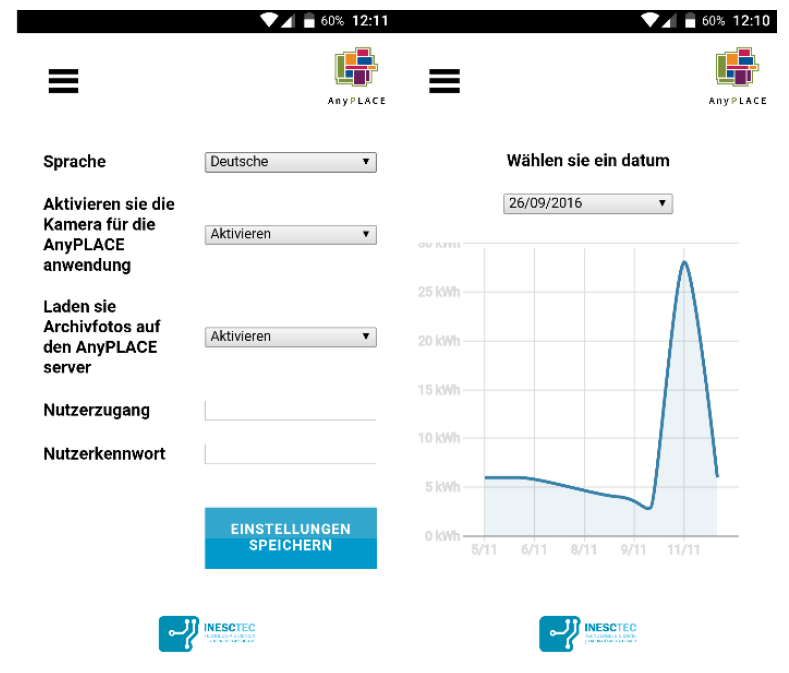

Fig. 1 Screenshots of AnyPLACE smartphone application

Besides being able to send energy metering reports to data servers, the AnyPLACE smartphone applications are also able to access the results from the energy management algorithms. Day-ahead schedules delivered by these algorithms are presented to the user, including appliance status and real-time control. Relying on graphical representation of the operating period of each appliance, the AnyPLACE smartphone application represents the usage of each appliance within a household, as can be seen on Fig. 2.

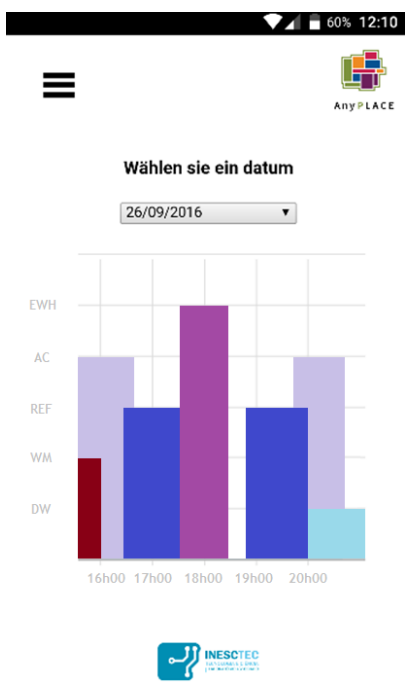

Fig. 2 Screenshots of AnyPLACE smartphone application

By accessing the day-ahead scheduling data stored by the AnyPLACE, smartphone applications can retrieve any information local operation data and delivering it to the users, regardless of their current location. Manual operation of appliances and overrule of day-ahead scheduling is also possible through these applications. Relying on a secure communication protocols such as HTTPS, along with encryption and tunneling techniques, any relevant data related to the AnyPLACE is capable of being changed in real-time and resilient to external tampering. Direct interaction between the AnyPLACE and appliances is dealt by the OpenHAB framework, which is detailed in section IV.

Integration of the multi-purpose GUI into the AnyPLACE EMS was accomplished by using an available open framework, which fulfilled requirements such as straight-through system integration, compatibility with Unix-based operating systems and adequate system performance. To achieve this, Qt framework [7] was chosen due to its versatility and widely available support, while being able to complete any other previous requirement.

Regarding the data storage required for the AnyPLACE applications, an evaluation of database solutions was carried out. To analyze the performance of currently available database solutions such as MySQL, PostgreSQL or SQLite, specific evaluation tests were conducted. Since PostgreSQL delivered the lowest memory consumption and CPU usage for intensive data operations, it was the selected candidate.

\section{ENERGY MANAGEMENT AlgORITHMS}

Demand response techniques for residential consumers usually aim at modulating the energy consumption towards a specific objective, either by changing its consumption (usually 
by reducing it) and/or by shifting consumption to a more favourable period. The energy management algorithms described in this section make use of potential flexibility of in the use of energy in domestic environments to pursue both energy efficiency and support DR strategies. Metering allows end-users to be aware of the consumption patterns and potential changes towards energy conservation and savings (behavioural approach). Through automation schemes the remote control and management of domestic appliances is possible per user preferences (technical approach). The goal of the technical approach is to allow energy and cost savings due to the flexibility provided to support the electricity system operation, taking advantage of the control of microgeneration, appliances, and energy storage units and at same time make use of smart plugs to control components that are not interoperable.

A set of algorithms were developed, to be integrated in the AnyPLACE platform, with the capability of performing a day ahead optimal scheduling of the home appliances. The energy management system will analyse, collect data and compare costs, providing energy awareness to the consumers from an active monitoring and control of appliances.

The most complex and relevant part of these algorithms is related to the optimal scheduling of flexible devices for the case of thermal appliances. For the AnyPLACE platform, a solution was specifically created for the home domain using mixed integer programming techniques to optimize the energy use. Analysing consumption patterns, through automated detection schemes, and considering the characteristics of appliances and the user preferences, the algorithms will be able to make an optimal scheduling for all the connected appliances that can be classified as thermal and shiftable, regarding dynamic prices. The models of these appliances are respectively based in historical data and the physically-based models. The physically-based load models are used to characterize the behaviour of residential thermostatically controlled loads [8].

Shiftable appliances operate on a predefined cycle with a known duration and consumption. The idea is to shift their operation to periods where the price is lower. The configuration considers a deadline that is defined by the end-user, as far ahead as possible, with a maximum span of operation of $24 \mathrm{~h}$. A time window is available between a configuration time and deadline, during which the control method can choose the optimal start time.

The optimized consumption of thermal appliances can be obtained through an external on/off control schedule performed by the AnyPLACE EMS. The actual control temperatures are no longer the thermostat dead-band as in baseline. Instead, it is asked to the end-user to define in the AnyPLACE GUI the admissible comfort limits, within which the temperature should be kept by the control system.

An example of an optimization performed by the AnyPLACE platform, is presented in the objective function (1), where the aim is to find the lowest daily cost for the energy use. It is constrained by various factors: physical characteristics of appliances, end-user preferences and power limitations. $\min \left(\sum_{t=0}^{n s t e p s} C_{t} *\left[P_{t}^{s}+P_{t}^{n s}\right]\right)$

$P_{t}^{s}=\sum_{j=1}^{n} \sum_{i=0}^{d_{j}}\left[P_{i}^{j} \times \lambda_{t-i}^{j}\right]$

$P_{t}^{n s}=\sum_{k=1}^{m}\left[P^{m} \times \lambda_{t}^{m}\right]$

Where,

$\boldsymbol{n}_{\text {steps }}$, number of control periods

$\boldsymbol{n}$, number of shiftable appliances

$\boldsymbol{d}_{\boldsymbol{j}}$, duration of operation of appliance $j$

$\boldsymbol{P}_{\boldsymbol{i} \boldsymbol{j}}$, Power in period $i$ of appliance $j$

$\lambda_{t-i}^{j}$, binary variable-condition if appliance start in period $t-i$

$\boldsymbol{P}_{M C t}^{\boldsymbol{m}}$, binary variable-condition if appliance is operating or not

$\boldsymbol{m}$, number of thermal appliances

$\boldsymbol{P}^{\boldsymbol{m}}$, capacity of thermal appliance $m$ in $(\mathrm{kW})$

To create an example scenario to test the algorithm, the Spanish dynamic tariff [9] was used. Also, public data sets [10] were used to model shiftable appliances, and standard values for the parameters of thermal appliances were considered. The previous formulation was implemented in $\mathrm{C}++$ language to allow a performed-driven implementation. To solve the optimization problem stated above, two different solvers were considered and tested: GLPK [10] and SCIP [12]. The selection was limited to these two as they represent the very few open source implementations available to be used in an ARM-based single board computer, which is the processing module of the AnyPLACE solution.

To test the optimization formulation a specific problem was created, representing a typical house, where 6 appliances were considered. Since the control sample time was established at 15 min this means that 96 variables were created for each of the appliances to account for the intertemporal optimization of the day-ahead optimal scheduling. As such a total of 576 binary variables were defined (that accounted for the different activation and deactivation of each of the appliances) and 579 constraints (linear) and an optimal solution was produced with a 5\% gap (difference between the best found primal solution and the best possible solution) in a Raspberry Pi 2 on different times with both solvers. The result of this simulation was the cost optimized scheduling of appliances. For the same formulation, the SCIP solver achieved the same results taking roughly 11 times less than the GLPK. In a raspberry Pi 2 the optimization ran in 1 h09 whereas the SCIP only took $6 \mathrm{~m} 20$.

The example output, presented in Fig. 3 and Fig. 4, represents the difference between the normal work (baseline without optimization) and an optimized schedule (modified), in a household during a 24-hour interval. Fig. 5 represents the same cost optimized scenario but now with a maximum power limitation. This limitation represents an addition of 96 constraints to the optimization problem.

The normal operating conditions (Fig. 3 - baseline scenario) shows the result of a daily operation of the appliances (their activation) in a so-called typical day. Fig. 4 represents the 
modified appliances consumption, because of the optimization algorithm described above, which determined the best operating time when the Spanish dynamic tariff price curve is considered. Finally, Fig. 5 represents the result of the optimization algorithm with an additional constraint that limits the maximum power consumption in each time step, which works as power cap. This constraint is very relevant since in some European countries there is a limitation of the instantaneous power that can be drawn from the grid. This represents an additional cost in the electricity bill and it is usually associated to discrete power interval values. In these new scenarios, no restriction is violated and as such the appliances operation is not compromised

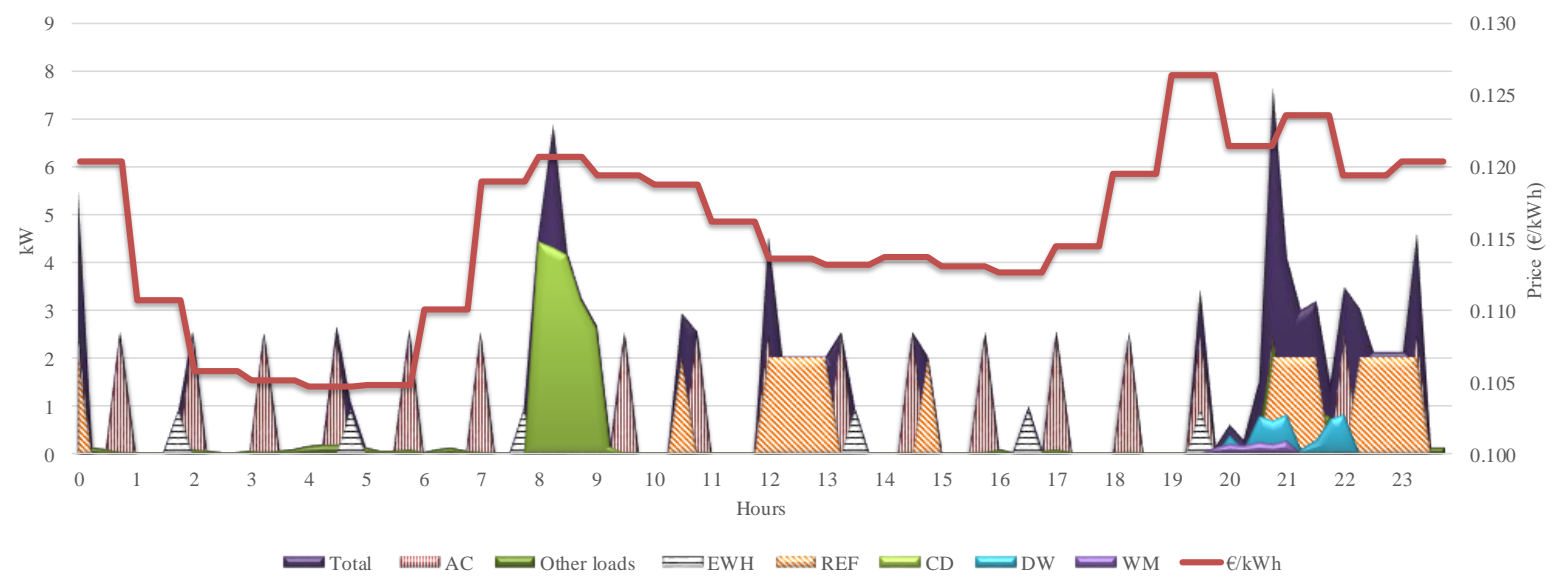

Fig. 3 - Baseline profile of all appliances regarding dynamic tariffs

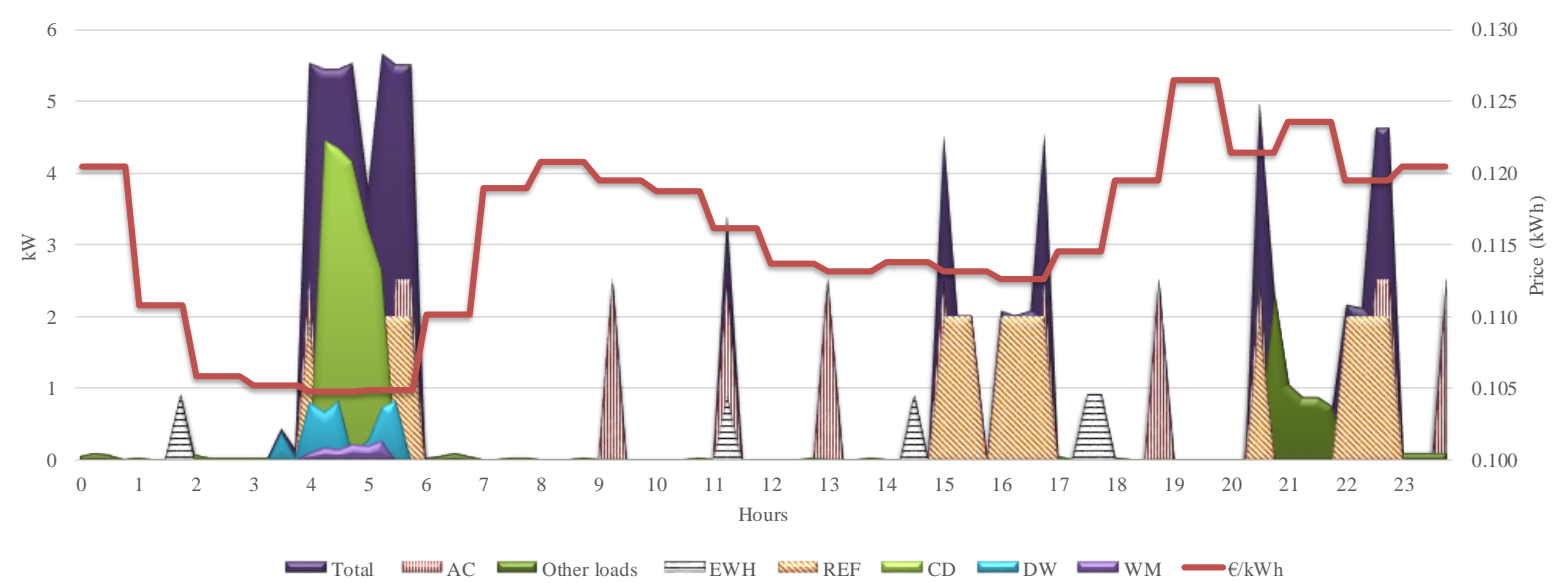

Fig. 4 - Optimized profile of all appliances regarding dynamic tariffs

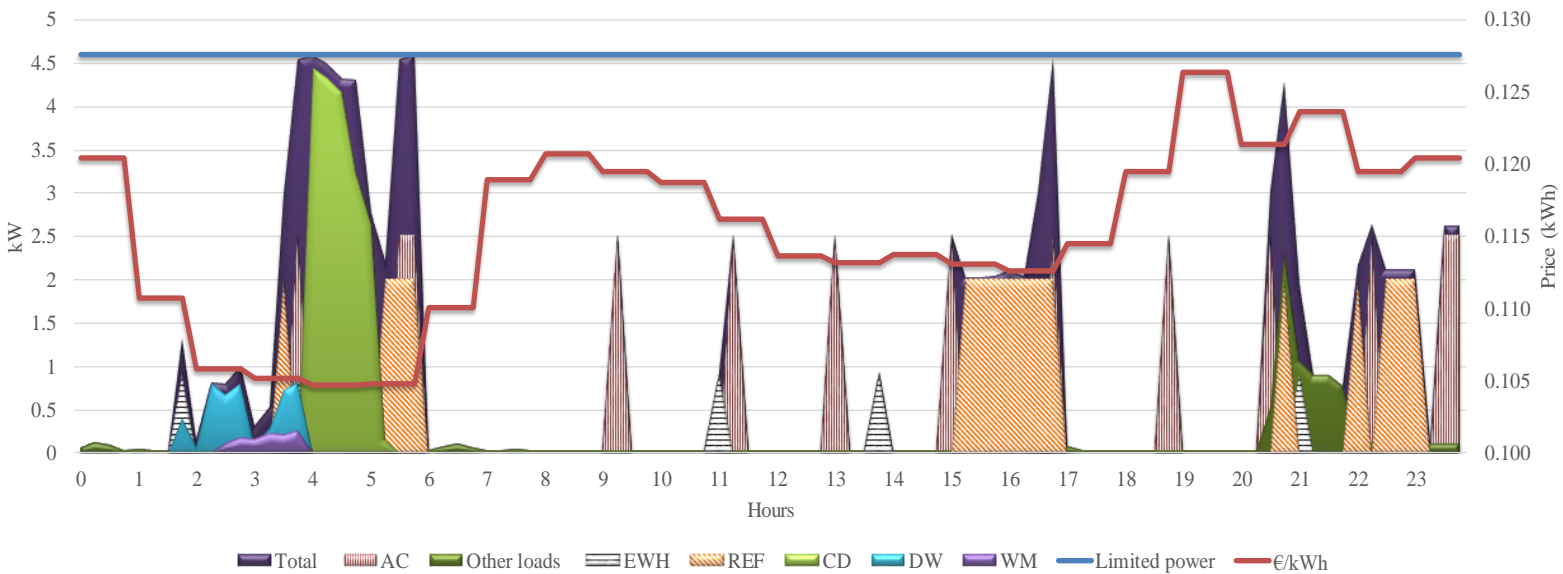

Fig. 5 - Optimized profile of all appliances regarding dynamic tariffs and limited power 
The automation implementation of the scheduling outputs from the energy management algorithm is defined and analysed in the next section.

\section{AUTOMATION SUPPORT}

A desirable characteristic of an energy management system is the ability to schedule the operation of existing appliances (and microgeneration, if available), which can be programmed manually or automatically. The scheduling of appliances is one of the outputs of the energy management algorithms and its implementation needs to take into consideration the type of appliance or system to be controlled.

Ideally the remote control of appliance and systems should be performed in a seamless way, with the informed consent of the end-user, but without the explicit need for their activation. The main challenge is related to the fact that current appliances are not prepared to be remotely configured by a central platform like AnyPLACE. Although some appliances do allow a remote operation via local communications or via a cloud service, most of them still relies on user interaction to schedule their operation.

An alternative being explored is the use of remote plugs (also known as smart plugs) that allow the activation of appliances, but limiting the control states to either on or off. Furthermore, not all appliances will be able to be controlled this way since they may require a manual activation (ex: rearming after disconnection) or because a prolonged disconnection may lead to an undesirable state of operation (e.g., restart). The energy management algorithms need to account for all of these restrictions in terms of monitoring and control to produce optimized and realistic scheduling results.

From a low-level automation perspective, the implementation of the scheduling outputs from the energy management algorithm must deal with several communication challenges. There may be different communication technologies already present in a house (e.g., ZigBee, Bluetooth or Wi-Fi) or used by appliances/systems that are capable of some sort of communication (e.g., smart appliances and smart plugs). Regardless of the type of device, the low-level automation platform must be able to receive feedback information from it and to transmit the desired information to it to implement the energy management schemes. To meet this challenge and seamlessly integrate all the devices in the house, several frameworks were evaluated considering licensing, interoperability, maintainability and modularity aspects as well as the ability to integrate the largest number of different technologies and devices.

The OpenHAB framework was chosen handle the data exchange in AnyPLACE EMS automation. It is based on the eclipse smart home framework and provides an interoperable platform that can connect to various smart devices from several manufacturers and allows creating automation rules to interconnect them.

The OpenHAB distinguishes between the device physically connected to the platform and the application logic (i.e. the information model). In the information model, physical devices or systems are represented by Things that provide many functionalities. Things have associated Channels that represent specific functions that are linked to items. Items represent (finegrained) functionality that is used by applications like user interfaces or automation logic. They define e.g. the type, name and state of Things channels. Links are the representation of communication technologies or protocols associated to Things [13]. The connection between the items and the channels of a thing is established by Bindings - optional interfaces used to extend the functionality of the platform [14].

Using OpenHAB different devices can be connected to the AnyPLACE EMS like smart appliances, smart plugs, PV inverters, smart lighting systems among many other devices. Variables like power consumption, energy production and lighting levels can be monitored by the EMS and used by the optimization algorithm to produce schedules according to an objective function. In Fig. 4 is presented a snippet representation of items for Philips Hue Lighting System in OpenHAB framework.

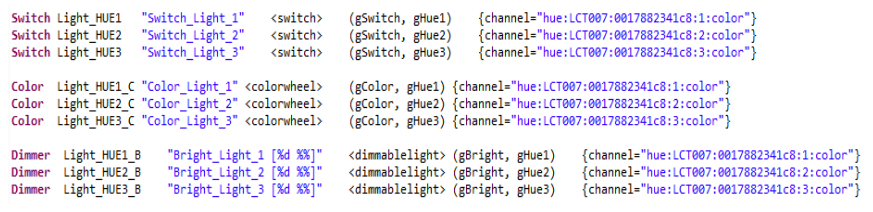

Fig. 6 OpenHAB representation

OpenHAB also provides an integrated web application interface where connected devices can be monitored and controlled from any screen within the home network using a simple browser. In Fig. 7 an example of a openHAB control and monitoring web interface featuring the DSP-W215 D-Link Smart Plug where it is possible to control its on/off state, monitor the instantaneous power consumption of the device connected through it, monitor the total energy amount consumed since the plug was connected and also the internal temperature of the D-Link device.

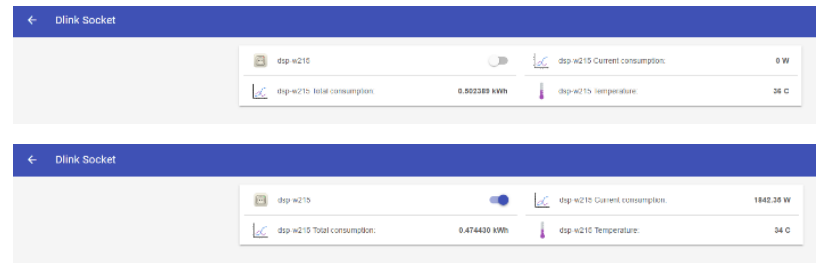

Fig. 7 - Representation of the openHAB web interface featuring D-Link DSP-W215 Smart Plug

\section{CONSIDERATIONS}

The energy management in houses can largely benefit from the use of solutions like the one being developed in the AnyPLACE project. The design of proper user interfaces, flexible and adaptable, to the needs of its used is a significant concern in providing the necessary engagement that is likely to produce long lasting energy efficiency behaviours.

This paper introduced the AnyPLACE platform which accounts for an adaptable GUI and automation framework that allow the implementation of energy management algorithms. These can be used to provide benefits to consumers, as it was shown, in allowing them to take advantage of favorable periods to allocate (schedule) their loads without compromising their 
comfort levels. The presented results showed that this kind of solutions can have the necessary intelligence to characterize the energy use of an entire house and produce optimized schedules for variable components (e.g., energy consumption) and fixed components (e.g., contracted power / power cap).

\section{REFERENCES}

[1] M. Parvania, M. Fotuhi-Firuzabad and M. Shahidehpour, "Optimal Demand Response Aggregation in Wholesale Electricity Markets," IEEE Transactions on Smart Grid, vol. 4, no. 4, pp. 1957-1965, December 2013.

[2] M. Heleno, M. A. Matos, J.A. Peças Lopes, “A bottom-up approach to leverage the participation of residential aggregators in reserve services markets", Electric Power Systems Research, vol. 136, pp. 425-433, July 2016.

[3] K. S. Vallerio, L. Zhong, and N. K. Jha, "Energy-Efficient Graphical User Interface Design," IEEE Transactions on Mobile Computing, vol. 5, no. 7, pp. 846-859, July 2006.

[4] R. Luostarinen, J. Manner, J. Maatta, R. Jarvinen, "User-centered design of graphical user interfaces," Military Communications Conference MILCON, pp. 50-55, August 2010.

[5] J. V. Bergh, K. Coninx, "Towards Integrated Design of ContextSensitive Interactive Systems," Proceedings of the $3^{\text {rd }}$ International
Conference on Pervasive Computing and Communications Workshops, pp-30-34, March 2005.

[6] Introduction to PhoneGap. Available: http://phonegap.com/getstarted/

[7] Qt for Application Development. Available: http://www.qt.io/qt-forapplication-development/

[8] M. L. D. Heleno, Ph.D. Thesis, "Provision of Advanced Ancillary Services Through Demand Side Integration.”. 2016. [Online]. Available: https://repositorio-aberto.up.pt/bitstream/10216/82179/2/130410.pdf

[9] Precio voluntario para el pequeño consumidor (PVPC) [Online]. Available: https://www.esios.ree.es/es/pvpc.

[10] O. Parson, "Disaggregated Homes," [Online]. Available: http://blog.oliverparson.co.uk/2012/06/public-data-sets-for-nialm.html

[11] GLPK (GNU linear programming kit), 2006.

[12] G. Gamrath, T. Fischer, T. Gally, A. M. Gleixner, G. Hendel, T. Koch, S. J. Maher, M. Miltenberger, B. Müller, M. E. Pfetsch, C. Puchert, D. Rehfeldt, S. Schenker, R. Schwarz, F. Serrano, Y. Shinano, S. Vigerske, D. Weninger, M. Winkler, J. T. Witt, J. Witzig, "The SCIP Optimization Suite 3.2". 2016. [Online] Available: https://opus4.kobv.de/opus4-zib/frontdoor/index/index/docId/5767

[13] OpenHAB Concepts. Available: http://docs.openhab.org/concepts/

[14] OpenHAB Bindings: Available:

https://github.com/openhab/openhab/wiki/Bindings

(C) 2017 IEEE. Personal use of this material is permitted. Permission from IEEE must be obtained for all other uses, in any current or future media, including reprinting/republishing this material for advertising or promotional purposes, creating new collective works, for resale or redistribution to servers or lists, or reuse of any copyrighted component of this work in other works. 